\title{
A Korea-Japan planet search program: Current status and discovery of a brown dwarf candidate
}

\author{
Masashi Omiya ${ }^{1}$, Hideyuki Izumiura ${ }^{2}$, Bun'ei Sato ${ }^{3}$, Michitoshi \\ Yoshida $^{2}$, Eiji Kambe ${ }^{2}$, Eri Toyota ${ }^{4}$, Seitaro Urakawa ${ }^{5}$, Seiji Masuda ${ }^{6}$, \\ Masahide Takada-Hidai ${ }^{7}$, Inwoo $\mathbf{H a n}^{8}$, Kang-Min $\mathbf{K i m}^{8}$, \\ Byeong-Cheol Lee ${ }^{8,9}$ and Tae Seog Yoon ${ }^{9}$ \\ ${ }^{1}$ Department of Physics, Tokai University, Hiratsuka, Kanagawa 259-1292, Japan \\ email: ohmiya@peacock.rh.u-tokai.ac.jp \\ ${ }^{2}$ Okayama Astrophysical Observatory, National Astronomical Observatory of Japan, Asakuchi, \\ Okayama 719-0232, Japan \\ ${ }^{3}$ Global Edge Institute, Tokyo Institute of Technology, Meguro-ku, Tokyo 152-8550, Japan \\ ${ }^{4}$ Graduate School of Science, Kobe University, Kobe, Hyogo 657-8501, Japan \\ ${ }^{5}$ Japan Spaceguard Association, Shibuya-ku, Tokyo 151-0073, Japan \\ ${ }^{6}$ Tokushima Science Museum, Asutamu Land Tokushima, Itano-gun, Tokushima 779-0111, \\ Japan \\ ${ }^{7}$ Liberal Arts Education Center, Tokai University, Hiratsuka, Kanagawa 259-1292, Japan \\ ${ }^{8}$ Korea Astronomy and Space Science Institute, Daejeon 305-348, South Korea \\ ${ }^{9}$ Department of Astronomy and Atmospheric Sciences, Kyungpook National University, Daegu \\ 702-701, South Korea
}

\begin{abstract}
Since 2005, we have been carrying out a precise radial velocity survey of about 190 intermediate-mass $\left(1.5-5 M_{\odot}\right) \mathrm{G}$ and $\mathrm{K}$ giants at Bohyunsan Optical Astronomy Observatory $(\mathrm{BOAO})$ in Korea and Okayama Astrophysical Observatory (OAO) in Japan, which aims to reveal statistical properties of planetary systems around intermediate-mass stars. We have finished the first screening of 120 stars so far and have identified 5 candidates with large periodic radial velocity variations. One of the candidates turned out to be orbited by a brown dwarf mass companion with minimum mass of $37.6 M_{J u p}$ and semimajor axis of 1.71 AU. The primary star has a mass of $3.9 M_{\odot}$, which ranks among the most massive stars with substellar companions. Our discovery may support the current view obtained from results of planet searches around intermediate-mass stars that massive substellar companions tend to form around massive stars.
\end{abstract}

Keywords. stars: low-mass, brown dwarfs, techniques: radial velocities

\section{Introduction}

To date, more than 200 exoplanets have been detected around solar-type stars by precise Doppler surveys. These surveys have unveiled various statistical characteristics of the planets. On the other hand, only 10 planets have been discovered around intermediatemass stars $\left(1.5-5 M_{\odot}\right)$ and their statistical properties have not been yet clear. Planetary systems around such massive stars are particularly important for constructing planet formation theories because they can constrain key processes of the planet formation such as dependence on host star's mass (probably related with mass of proto-planetary disk), role of radiation of central stars, and timescale of planet formation. However, early-type stars (B-A dwarfs), intermediate-mass stars on the main sequence, are not suitable for 
precise radial velocity survey due to fewer metallic absorption lines in their spectra and higher surface activity. Late $\mathrm{G}$ and early $\mathrm{K}$ type giants, intermediate-mass stars in the evolved stages, are promising targets for this purpose because they have many sharp absorption lines in their spectra and their surface activity is relatively low compared to early-type stars and more evolved late $\mathrm{K}$ and $\mathrm{M}$ type giants.

So far, radial velocity surveys of intermediate-mass stars (e.g. Johnson et al. 2006, Sato et al. 2007, Niedzielski et al. 2007) have revealed some properties of substellar companions around them. For example, frequency of massive substellar companions is higher than that of solar-type stars (Lovis \& Mayor 2007, Johnson et al. 2007), and many massive host stars have lower metallicity than typical one of solar-type host stars (e.g. Sato et al. 2003). These properties seem to be not similar to those of solar-type stars and such different properties should be explored by further studies. In the case of solar-type stars, a "brown dwarf desert" is widely known as a paucity of substellar companions falling in the brown dwarf mass region (13-80 $M_{J u p}$ ) (Marcy \& Butler 2000, Halbwachs et al. 2000, Grether \& Lineweaver 2006), which suggests distinct formation mechanisms between planets and stellar companions. To uncover whether a brown dwarf desert also exist around intermediate-mass stars will give us a clue to understand formation mechanism of planets around them.

\section{Korea-Japan Planet Search Program}

We are monitoring our targets using the $1.8 \mathrm{~m}$ telescope at BOAO with Bohyunsan Optical Echelle Spectrograph (BOES, $R=\lambda / \Delta \lambda=50000,3500 \AA-10500 \AA$ ), which is a fiberfed high resolution echelle spectrograph (Kim et al. 2007) and the $1.88 \mathrm{~m}$ telescope at

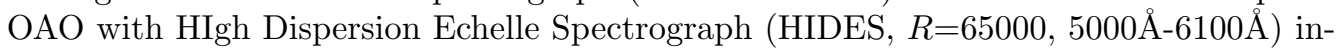
stalled at the coude focus of the telescope (Izumiura 1999). For precise radial velocity measurements, an iodine absorption cell (I2 cell) is installed at the optical path in front of the fiber or slit of each spectrograph (Kim et al. 2002, Kambe et al. 2002). We also take stellar spectra without I2 cell for abundance analysis. Radial velocities are derived by using Sato et al. (2002)'s code which models an I2-superposed stellar spectrum with high resolution stellar and iodine templates and reproduced instrumental profiles of the spectrographs. We have achieved long term radial velocity precisions of $\sim 11 \mathrm{~m} / \mathrm{s}$ and $\sim$

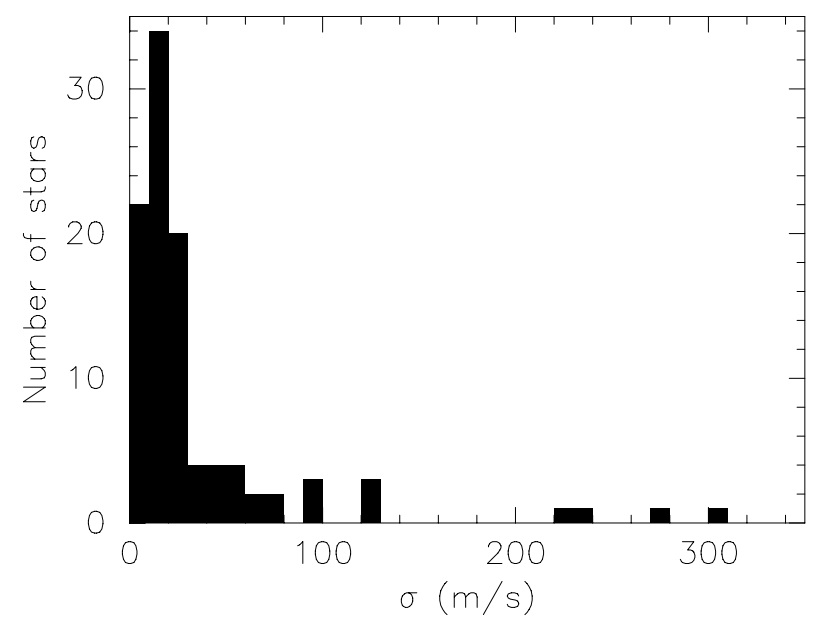

Figure 1. Velocity RMS of 116 BOAO \& OAO targets observed more than three times for 2.5 years. 
6-7 m/s with BOES and HIDES, respectively, over a time span of 2.5 years during the project. A Korea-Japan planet search program has been conducting a precise Doppler survey of about $190 \mathrm{G}$ and $\mathrm{K}$ giants at BOAO in Korea and OAO in Japan since 2005. This joint planet search program is an extended version of on-going OAO planet search program (Sato et al. 2005) and a part of the international collaborations between Korea, China and Japan (East-Asian Planet Search Network, EAPSNet, Izumiura 2005) aiming to obtain the properties of planetary systems around intermediate-mass stars by surveying a total of "1000" giant stars for planets.

\section{Current status and Orbital solution of a brown dwarf companion}

Up to now, we observed about 120 targets more than three times. Seventeen of the targets exhibited large radial velocity variations with amplitudes of $50 \mathrm{~m} / \mathrm{s}-350 \mathrm{~m} / \mathrm{s}$ and five of them showed probable periodic variations (See Figure 1).

One of such targets clearly shows large periodic radial velocity variations as seen in Figure 2. Low surface activity of this star favors an orbital motion as the cause of the observed variability. On the basis of Keplerian orbital fit, we obtained a velocity semiamplitude of $413.5 \pm 2.6 \mathrm{~m} / \mathrm{s}$, period of $410.5 \pm 0.6$ days and eccentricity of $0.082 \pm$ 0.007. Adopting a host star's mass of $3.9 \pm 0.4 M_{\odot}$, which was estimated from evolutionary track and fundamental stellar parameters of $L=251 \pm 95 \mathrm{~L}_{\odot}, T_{\text {eff }}=5083 \pm 103$ $\mathrm{K}$, and $[\mathrm{Fe} / \mathrm{H}]=0.04 \pm 0.18$, we obtained a minimum mass for the companion $m_{p} \sin i=$ $37.6 \pm 2.6 M_{J u p}$ and a semimajor-axis of $a=1.71 \pm 0.06$ AU. If we assume the orbit is randomly oriented, there is a $12 \%$ chance that the true mass exceeds $80 M_{J u p}\left(i \geqslant 28^{\circ}\right)$.

\section{Summary}

We have been carrying out a precise redial velocity survey of $\mathrm{G}$ and $\mathrm{K}$ giants at BOAO and $\mathrm{OAO}$ and discovered a brown dwarf mass companion together with some planetary candidates so far. This brown dwarf companion is the forth one among those discovered around intermediate-mass giants. The host star has a mass of $3.9 M_{\odot}$, which is one of the most massive stars harboring substellar companions. Three stars with masses of larger

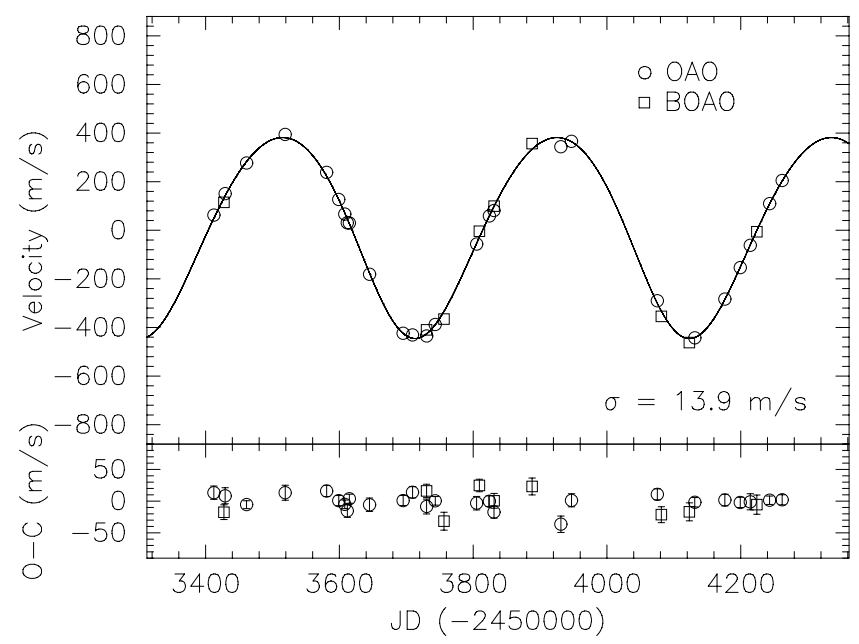

Figure 2. Radial velocities and orbital fit of a star with a brown dwarf candidate from BOAO (open squares) and $\mathrm{OAO}$ (open circles). O-C indicates difference between each observational point and orbital curve. 
than $3 M_{\odot}$ are currently known to harbor substellar companions (Hatzes et al. 2005, Lovis \& Mayor 2007, this work) and all the companions have brown dwarf mass. The result supports the current view that more massive substellar companions tend to form around more massive stars (Lovis \& Mayor 2007, Johnson et al. 2007).

So far, we have detected two brown dwarf mass companions (Liu et al. 2008 and this work) among a total of about 500 targets from OAO and BOAO-OAO surveys. The current detection rate of brown dwarf companion in our sample is thus estimated to be 0.4 $\%$, which seems to be comparable to that of less than $1 \%$ for solar-type stars (Marcy \& Butler 2000, Grether \& Lineweaver 2006). It is still unclear, however, whether a brown dwarf desert also exists among intermediate-mass stars. Since less massive planets are normally difficult to detect around evolved giants due to their larger intrinsic radial velocity variability (up to $10-20 \mathrm{~m} / \mathrm{s}$ ), a mass distribution of planetary mass companions around them has not been well established yet. Further investigation of a mass distribution of substellar companions is required to elucidate formation mechanism of planets and brown dwarfs around massive stars.

\section{References}

Grether, D., \& Lineweaver, C. H. 2006, ApJ, 640, 1051

Halbwachs, J. L., Arenou, F., Mayor, M., Udry, S., \& Queloz, D. 2000, A\&AA, 355, 581

Hatzes, A. P., Guenther, E. W., Endl, M., Cochran, W. D., Döllinger, M. P., \& Bedalov, A. 2005, A\&BA, 437, 743

Izumiura, H. 1999, in Proc. 4th East Asian Meeting on Astronomy, ed. P.S. Chen (Kunming: Yunnan Observatory), 77

Izumiura, H. 2005, Journal of Korean Astronomical Society, 38, 81

Johnson, J. A., Marcy, G. W., Fischer, D. A., Henry, G. W., Wright, J. T., Isaacson, H., \& McCarthy, C. 2006, ApJ, 652, 1724

Johnson, J. A., Butler, R. P., Marcy, G. W., Fischer, D. A., Vogt, S. S., Wright, J. T., \& Peek, K. M. G. 2007, ApJ, 670, 833

Kambe, E., Sato, B., Takeda, Y., Ando, H., Noguchi, K., Aoki, W., Izumiura, H., Wada, S., Masuda, S., Okada, N., Shimizu, Y., Watanabe, E., Yoshida, M., Honda, S., Kawanomoto, S. 2002, PASJ, 54, 865

Kim, K.-M., Jang, B.-H., Han, I., Jang, J. G., Sung, H. C., Chun, M.-Y., Hyung, S., Yoon, T.-S., Vogt, S. S. 2002, Journal of Korean Astronomical Society, 35, 221

Kim, K. M., Han, I., Valyavin, G., Plachinda, S., Jang, J. G., Jang, B. -H., Seong, H. C., Kang, D. I., Park, B. G., Yoon, T. S. and Vogt, S., 2007, PASP, 119, 1052.

Liu, Y. J., Sato, B., Zhao, G., Noguchi, K., Wang, H., Kambe, E., Ando, H., Izumiura, H., Chen, Y. Q.; Okada, N., Toyota, E., Omiya, M., Masuda, S., Takeda, Y., Murata, D., Itoh, Y., Yoshida, M., Kokubo, E., \& Ida, S., 2008, ApJ, 672, 553

Marcy, G. W., \& Butler, R. P. 2000, PASP, 112, 137

Niedzielski, A., Konacki, M., Wolszczan, A., Nowak, G., Maciejewski, G., Gelino, C. R., Shao, M., Shetrone, M., \& Ramsey, L. W. 2007, ApJ, 669, 1354

Sato, B., Kambe, E., Takeda, Y., Izumiura, H., \& Ando, H. 2002, PASJ, 54, 873

Sato, B., Ando, H., Kambe, E., Takeda, Y., Izumiura, H., Masuda, S., Watanabe, E., Noguchi, K., Wada, S., Okada, N., Koyano, H,. Maehara, H., Norimoto, Y., Okada, T., Shimizu, Y., Uraguchi, F., Yanagisawa, K., \& Yoshida, M. 2003, ApJ, 597, L157

Sato, B., Kambe, E., Takeda, Y., Izumiura, H., Masuda, S., \& Ando, H. 2005, PASJ, 57, 97

Sato, B., Izumiura, H., Toyota, E., Kambe, E., Takeda, Y., Masuda, S., Omiya, M., Murata, D., Itoh, Y., Ando, H., Yoshida, M., Ikoma, M., Kokubo, E., \& Ida, S. 2007, ApJ, 661, 527 